\title{
Effect of Uniaxial Pressure on the Ferroelectric Phase Transition in $\mathrm{Pb}_{5} \mathrm{Ge}_{3} \mathrm{O}_{11}$ : $\mathrm{Ba}$ Crystals
}

\author{
A. $\operatorname{Molak}^{a}$, M. Koralewski ${ }^{b, *}$, G.A. Saunders ${ }^{c}$ \\ AND W. JUSZCZYK ${ }^{a}$ \\ ${ }^{a}$ Institute of Physics, University of Silesia \\ Uniwersytecka 4, 40-007 Katowice, Poland \\ ${ }^{b}$ Institute of Physics, A. Mickiewicz University \\ Umultowska 85, 61-614 Poznań, Poland \\ ${ }^{c}$ Department of Physics, University of Bath \\ Claverton Down, Bath, BA2 7AY England
}

(Received March 29, 2005)

\begin{abstract}
The effect of uniaxial pressure up to $1 \mathrm{kbar}$ applied perpendicular and parallel to $c$-axis, on the Curie temperature and electric permittivity $\varepsilon_{c}$ of Ba-doped $\mathrm{Pb}_{5} \mathrm{Ge}_{3} \mathrm{O}_{11}$ single crystals was investigated. The Curie temperature decreases with uniaxial pressure and the coefficient $\gamma_{i}\left(\gamma_{i} \equiv \delta T_{\mathrm{c}} / \delta X_{i}\right)$ is determined as -7 and $-11 \mathrm{~K} \mathrm{kbar}^{-1}$ for the $b$ - and $c$-axes, respectively. It was found that uniaxial pressure increases the phase transition diffuseness and the tricritical point cannot be achieved for this pyroelectric and ferroelectric single crystal.
\end{abstract}

PACS numbers: 77.80.Bh, 77.84.Bw

\section{Introduction}

Lead germanate $(\mathrm{LGO})-\mathrm{Pb}_{5} \mathrm{Ge}_{3} \mathrm{O}_{11}$ is an important oxide ferroelectric and pyroelectric crystal on account of its application as the sensing material in infrared imaging and electrooptical devices [1-4]. In consequence, its optical properties are

*corresponding author; e-mail: koral@amu.edu.pl 
of great interest: recently, the photorefractive effects in undoped and Fe- and Rh-doped LGO crystals and lead barium $\mathrm{Pb}_{x} \mathrm{Ba}_{x} \mathrm{Ge}_{3} \mathrm{O}_{11}$ have been investigated [5].

LGO undergoes a second order phase transition $(\mathrm{PT})$ at $T_{\mathrm{c}} \approx 450 \mathrm{~K}$ from its paraelectric phase (hexagonal space group $P \overline{6}$ ) to its ferroelectric phase (trigonal space group P3) [6]. A small amount of an appropriate metal ion additive significantly affects the crystallization process and physical properties of LGO crystals $[5,6]$. Generally as the metal ion dopant concentration is increased, the Curie temperature $\left(T_{\mathrm{c}}\right)$ decreases. This influence of dopant is quite similar to the effects of hydrostatic $(p)$ or uniaxial pressure $(X)$ on the $T_{\mathrm{c}}$ of pure LGO single crystal [6].

Systems for which the line of the first order phase transition develops into that of the second order one, in the thermodynamical space of three independent fields, e.g. pressure ( $p$ or $X)$, temperature $(T)$, and electric field $(E)$, are known to show a tricritical point (TCP) [7]. Within Landau's phenomenological theory, the TCP is realized when the coefficient of the fourth power of polarization is equal to zero (i.e. $B=0$ ). As well as such a specific value of a critical coefficient, another indication of the TCP in the case of ferroelectric crystals is the ratio of slopes of $\varepsilon^{-1}$ versus $T$ below and above the transition, which at the TCP should be close or equal to the value of 4 [8]. The occurrence of a TCP has been established in a few ferroelectrics (for example, see Ref. [9]). In the case of oxide ferroelectrics, the TCP has been described only in $\mathrm{BaTiO}_{3}$ in which it is induced by application of a hydrostatic pressure of about $35 \mathrm{kbar}$ [10]. This high-pressure range requires the use of non-standard measuring chambers for dielectric measurements so that such studies are rarely performed. Some previous work on ferroelectrics [9] suggests that the TCP can be induced by uniaxial pressure of one order of magnitude lower than the required hydrostatic pressure.

A possible but not conclusive indication of the TCP induced under the influence of hydrostatic pressure of about $10 \mathrm{kbar}$ has been reported for pure [11] and Ba-doped [12] LGO crystals. Recent studies of the effect of uniaxial pressure on the ferroelectric phase transition of LGO single crystal suggests that it could be possible to induce the tricritical point by this approach [13]. Moiseenko et al. [14] reported a jump-like change in the hyper-Rayleigh light scattering at $T_{\mathrm{c}}=438 \mathrm{~K}$ in the LGO crystals slightly doped with lanthanum. Their results may indicate that the phase transition, in those crystals, may be close to a first order one. These examples suggest LGO or doped LGO as a promising material for model analysis of TCP within the group of oxide ferroelectric crystals.

In the present work the effect of uniaxial pressure on the ferroelectric phase transition in $\mathrm{Pb}_{5-x} \mathrm{Ba}_{x} \mathrm{Ge}_{3} \mathrm{O}_{11}$ (LGO:Ba) $(x=0.25)$ single crystal was studied.

\section{Experimental details}

The single crystals of LGO:Ba were grown using the Czochralski technique. The boule, transparent light yellow in colour, was pulled along the $c$-axis and 
had diameter of about $20 \mathrm{~mm}$. The Laue photographs were taken on a $c$-plate at room temperature to check the position of the $c$-axis. Samples in the form of small parallelepipeds of approximately $0.6 \times 0.5 \times 1.2 \mathrm{~mm}^{3}$ were cut from a well oriented $c$-plate. Uniaxial compression in the range up to 1000 bar was applied to these bar samples using a lever and a weight device. The measured accuracy of compression was estimated as about 10 bar. Sample capacitance $(C)$ and the conductance $(G)$ were recorded under constant pressure, at frequencies of $0.1,1$, 10, 20, and $100 \mathrm{kHz}$ using a HP 4263B LCR meter. Temperature was varied within the range 300 to $500 \mathrm{~K}$, at a rate $1 \mathrm{~K} / \mathrm{min}$ in subsequent cycles of heating and cooling. Other details of experiment were the same as described previously [13].

\section{Results and discussion}

The temperature dependence of the electric permittivity along the $c$-axis $\left(\varepsilon_{\mathrm{c}}\right)$ of pure $\mathrm{Pb}_{5} \mathrm{Ge}_{3} \mathrm{O}_{11}$ and Ba-doped, $\mathrm{Pb}_{4.75} \mathrm{Ba}_{0.25} \mathrm{Ge}_{3} \mathrm{O}_{11}$ crystals at zero uniaxial pressure was measured. The maximum value $\varepsilon_{\max }$ of $\varepsilon_{c}$ at the transition temperature is $\approx 3400$ for $100 \mathrm{kHz}$ for pure LGO crystal and decreases to $\approx 750$ for the Ba-doped crystal. The transition temperature $T_{\mathrm{c}}$, determined as the temperature at which the electric permittivity reaches its maximum, depends weakly on the measuring frequency and increases by only $0.5 \mathrm{~K}$ in the applied frequency range $100 \mathrm{~Hz}$ to $100 \mathrm{kHz}$. Dispersion of $\varepsilon_{\max }$ was observed both in the ferroelectric and paraelectric phases $[13,15]$. The values of $\varepsilon_{c}$ obtained both at room temperature or near the phase transition temperature for the two crystals studied change slightly depending on previous thermal and mechanical treatment of the chosen samples.

In the case of pure LGO crystal the reciprocal of the electric permittivity $\varepsilon_{c}$ obeys the Curie-Weiss law $[6,13]$. The Curie-Weiss temperature $T_{0}$ is about $2.5^{\circ} \mathrm{C}$ lower than $T_{\mathrm{c}} \approx 450 \mathrm{~K}$ for these crystals. The Curie-Weiss constant $C^{+}$in the paraelectric phase, determined at $f=2 \mathrm{kHz}$, is equal to $C^{+}=1.15 \times 10^{4} \mathrm{~K}$ for the pure crystals (see Table). These values are in quite good agreement with those reported by Iwasaki et al. [16] and Namamatsu et al. [17] for pure LGO. The ratio of the slopes of $\varepsilon^{-1}$ against $T$ below and above the transition $\left(1 / C^{-}\right) /\left(1 / C^{+}\right)=$ $C^{+} / C^{-}$was calculated to be 2.4 (at $f=2 \mathrm{kHz}$ ). Such values indicate that the

TABLE

Parameters describing ferroelectric properties of pure and Ba-doped LGO single crystals at ambient pressure $(X=0, f=100 \mathrm{kHz}$, cooling) and uniaxial pressure coefficient $\gamma_{i}$.

\begin{tabular}{c|c|c|c|c|c|c|c}
\hline \hline Crystal & $T_{\mathrm{c}}$ & $\varepsilon_{\max }$ & $\begin{array}{c}C \\
{\left[10^{4} \mathrm{~K}\right]}\end{array}$ & $\frac{C^{+}}{C^{-}}$ & $\xi$ & $\gamma \perp c$ & $\gamma \| c$ \\
\cline { 5 - 7 } & {$[\mathrm{K}]$} & & & \multicolumn{2}{|c|}{$\left[\mathrm{K} \mathrm{kbar}^{-1}\right]$} \\
\hline LGO & 450 & 3400 & $1.15 \pm 0.05$ & 2.4 & 1 & $-(7.0 \pm 0.3)$ & $-(3.2 \pm 0.3)$ \\
LGO:Ba & 366 & 750 & $1.70 \pm 0.05$ & 2.13 & $1.75 \pm 0.05$ & $-(7 \pm 1)$ & $-(11 \pm 2)$
\end{tabular}


paraelectric-ferroelectric PT is of second order for the crystal studied in agreement with earlier findings $[6,13]$.

Now, we turn to discuss the results obtained for $\mathrm{Pb}_{4.75} \mathrm{Ba}_{0.25} \mathrm{Ge}_{3} \mathrm{O}_{11}$ crystal. The dependence of the maximum value $\varepsilon_{\max }$ of $\varepsilon_{c}$ on the uniaxial pressure is shown in Fig. 1. For pressure applied parallel to the $c$-axis this maximum value $\varepsilon_{\max }$ at $T_{\mathrm{c}}$ increases with increasing uniaxial pressure to a very broad maximum at about 250 bar. For the pressure applied parallel to the $b$-axis increase in $\varepsilon_{\max }$ with increasing pressure is observed; such behaviour of $\varepsilon_{\max }(X)$ occurs independently of the frequency in the range $100 \mathrm{~Hz}$ to $100 \mathrm{kHz}$ studied. A permanent increase in $\varepsilon_{\max }(X)$ induced when pressure was applied along the $b$-axis differs from the dependence found in pure LGO crystals [13].

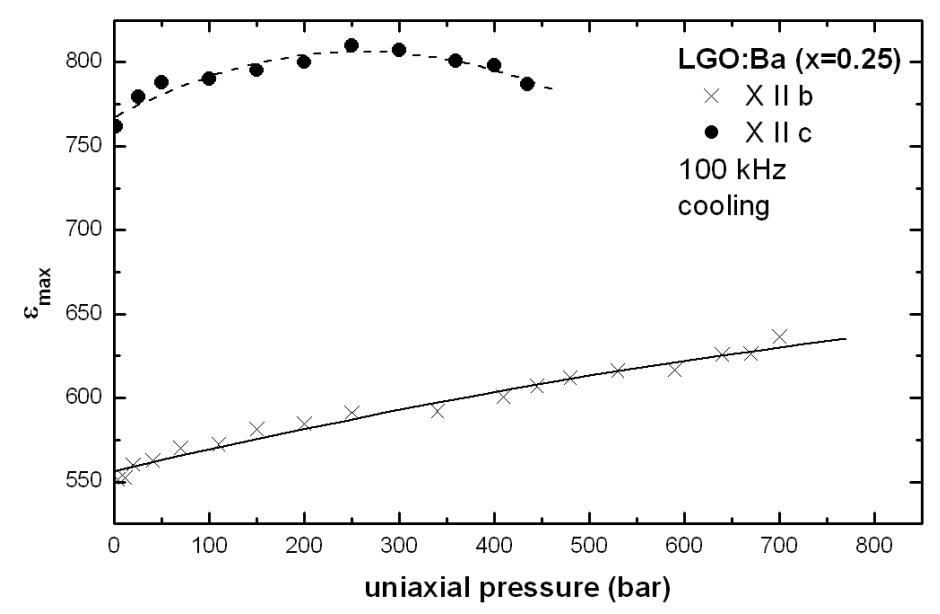

Fig. 1. The maximum value $\varepsilon_{\max }$ of the electric permittivity $\varepsilon_{c}$ as a function of uniaxial pressure applied along the $b$ - and $c$-axes measured for decreasing temperature for the $\mathrm{Pb}_{4.75} \mathrm{Ba}_{0.25} \mathrm{Ge}_{3} \mathrm{O}_{11}$ single crystal (frequency: $100 \mathrm{kHz}$ ).

The shifts of the phase transition temperature $T_{\mathrm{c}}^{X}$ induced by uniaxial pressure acting along the $b$ - and $c$-axes obtained from $\varepsilon\left(X_{i}, T\right)$ recorded at decreasing temperature (i.e. on cooling) are shown in Fig. 2. If this dependence is taken to be linear, the rate of decrease in the transition temperature can be expressed by the following equation:

$$
T_{\mathrm{c}}^{X}=T_{\mathrm{c}}^{0}+\gamma_{i} X_{i} \quad(i=b, c),
$$

where $T_{\mathrm{c}}^{X}$ and $T_{\mathrm{c}}^{0}$ are the transition temperatures at uniaxial pressure $X_{i}$ and atmospheric pressure, respectively $\left(T_{\mathrm{c}}^{0} \approx 366 \mathrm{~K}\right)$. The coefficients $\gamma_{i} \approx \delta T_{\mathrm{c}} / \delta X_{i}$ obtained from the best fit to the experimental isobaric points are collected in Table.

The $\varepsilon^{-1}(T)$ characteristic shows a diffused maximum and the applied uniaxial pressure further broadens the peak. Although the electric permittivity char- 


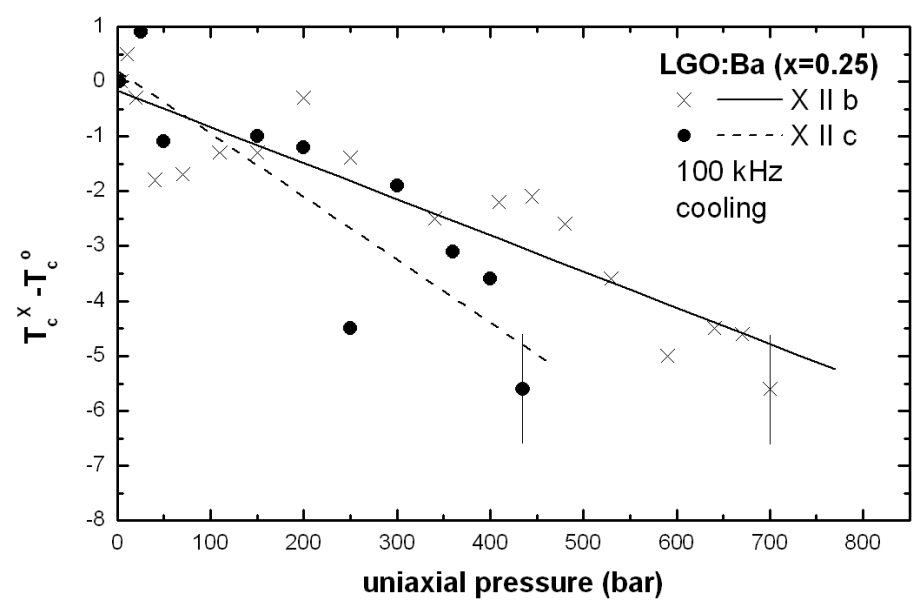

Fig. 2. Shift of the Curie point $\left(T_{\mathrm{c}}^{X}-T_{\mathrm{C}}^{0}\right)$ as a function of uniaxial pressure applied along the $b$ - and $c$-axes measured for decreasing temperature for the $\mathrm{Pb}_{4.75} \mathrm{Ba}_{0.25} \mathrm{Ge}_{3} \mathrm{O}_{11}$ single crystal. The lines represent linear fits. Frequency: $100 \mathrm{kHz}$; for each point error bars are the same as the examples shown.

acteristic is diffused, the Curie-Weiss temperature $\left(T_{0}\right)$ is only $3 \mathrm{~K}$ lower than $T_{\mathrm{c}}=T\left(\varepsilon_{\max }\right)$. That the values of $T_{\mathrm{c}}-T_{0}=3 \mathrm{~K}$ and $2.5 \mathrm{~K}$, obtained for the Ba-doped and pure LGO crystals, are almost the same suggests the second order nature of this phase transition is unchanged by doping with Ba ions.

Due to the wide, diffusive shape of the $\varepsilon(T, X)$ characteristic, the calculation of the ratio of the slopes of $\varepsilon^{-1}$ against $T$ below and above the transition $\left(1 / C^{-}\right) /\left(1 / C^{+}\right)=C^{+} / C^{-}$for different uniaxial pressure was not possible using the standard Curie-Weiss formula. Therefore, to describe the diffused $\varepsilon(T, X)$ characteristic around the phase transition the similar relation introduced by Santos and Eiras [18] was used

$$
\varepsilon=\varepsilon_{\max } /\left\{1+\left[\left(T-T_{\mathrm{m}}\right) / D\right]^{\xi}\right\} .
$$

Here, $\varepsilon_{\max }$ is the maximum of the real part of electric permittivity, $T_{\mathrm{m}}$ is the temperature corresponding to the maximum electric permittivity, and $D$ is a generalized Curie-Weiss constant. The parameter $\xi$ relates to the peak broadening of the anomaly in electric permittivity near the phase transition. The experimental data, $\varepsilon(T, X)$, obtained at subsequently applied, fixed pressures $X_{i}$, have been fitted with the use of Eq. (2) within the wide temperature range, $5 \mathrm{~K}<T-T_{\mathrm{m}}<95 \mathrm{~K}$. The uniaxial pressure dependence of the evaluated Curie-Weiss constant $C[\mathrm{~K}]$ vs. compression is plotted in Fig. 3. It turns out that the Curie-Weiss constant is independent of the uniaxial pressure within the experimental uncertainties in the pressure range studied.

The Curie-Weiss constant value $C^{+}=1.70 \times 10^{4} \mathrm{~K}$ obtained for the paraelectric phase of the $\mathrm{Pb}_{4.75} \mathrm{Ba}_{0.25} \mathrm{Ge}_{3} \mathrm{O}_{11}$ crystal (see Table) is in reasonable agree- 


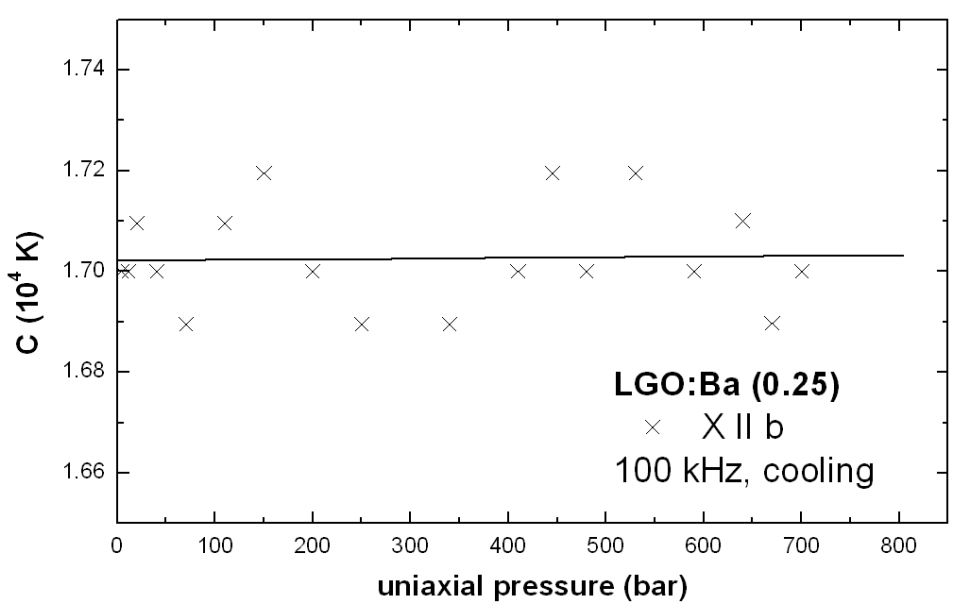

Fig. 3. The Curie-Weiss constant as a function of uniaxial pressure applied along the $b$-axis measured for decreasing temperature for the $\mathrm{Pb}_{4.75} \mathrm{Ba}_{0.25} \mathrm{Ge}_{3} \mathrm{O}_{11}$ single crystal (frequency: $100 \mathrm{kHz}$ ).

ment with that reported by Strukow et al. [19] for series of Ba-doped crystals. The ratio of the slopes of $\varepsilon^{-1}$ against $T$ below and above the transition $\left[\left(1 / C^{-}\right) /\left(1 / C^{+}\right)=C^{+} / C^{-}\right]$is calculated to be 2.13 , close to the value $C^{+} / C^{-}=2$ expected for a second order transition in the Landau model.

The parameter $\xi$, which describes diffuseness of a phase transition, was also calculated; its dependence on uniaxial pressure shows (Fig. 4) that diffusive character of the phase transition increases with applied uniaxial pressure.

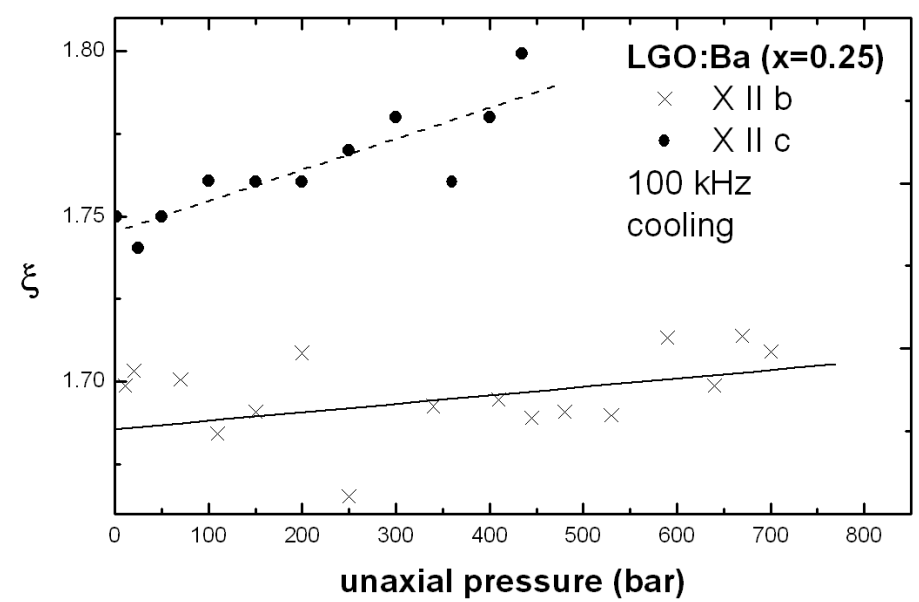

Fig. 4. The coefficient $\xi$ of Eq. (2) as a function of uniaxial pressure applied along the $b$ - and $c$-axes measured for decreasing temperature for the $\mathrm{Pb}_{4.75} \mathrm{Ba}_{0.25} \mathrm{Ge}_{3} \mathrm{O}_{11}$ single crystal (frequency: $100 \mathrm{kHz}$ ). 
There is an anisotropy of axial pressure influence on the electric properties of the $\mathrm{Pb}_{4.75} \mathrm{Ba}_{0.25} \mathrm{Ge}_{3} \mathrm{O}_{11}$ crystal in that the rate of change of parameter $\xi$ with uniaxial pressure applied along the $c$-axis is higher than that for pressure applied perpendicularly to the $c$-axis (see Fig. 4 ). The phase transition became diffuse both as a result of doping and application of pressure: TCP cannot be observed.

\section{Conclusions}

Our experiments show a linear change of $T_{\mathrm{c}}$ with uniaxial pressure up to 1 kbar applied along the $b$ - and $c$-axes of $\mathrm{Pb}_{5-x} \mathrm{Ba}_{x} \mathrm{Ge}_{3} \mathrm{O}_{11}$ (LGO:Ba) $(x=0.25)$. The negative values of $\gamma_{i}$ indicate the displacive character of the PT in the LGO-Ba doped single crystal in accordance with Samara's [20] empirical rules. The fit of the diffused $\varepsilon(T, X)$ characteristic with the use of Eq. (2) shows that the Curie-Weiss constant is not affected by the applied uniaxial pressure. It was found that application of uniaxial pressure perpendicular and parallel to the $c$-axis of LGO:Ba single crystal makes the phase transition diffuse. The diffusive character of PT is enhanced as uniaxial pressure is increased so that TCP cannot be achieved.

\section{References}

[1] H.H. Otto, P. Loster, Ferroel. Lett. 16, 81 (1993).

[2] G.R. Jones, N. Shaw, A.E. Vere, Electron. Lett. 8, 345 (1972).

[3] R. Watton, C. Smith, G.R. Jones, Ferroelectrics 14, 917 (1976).

[4] W. Eysel, R.W. Wolf, R.E. Newnham, J. Am. Ceram. Soc. 56, 185 (1973).

[5] X. Yue, S. Mendricks, Y. Hu, H. Hesse, D. Kip, J. Appl. Phys. 83, 3473 (1998).

[6] Landolt-Börnstein, New Series, Group III, Vol. 16a, Ferroelectrics and Related Substances: Oxides, Ed. K.-H. Hellwege, Springer, Berlin 1982, p. 244.

[7] R.B. Griffiths, Phys. Rev. Lett. 24, 715 (1970).

[8] L.D. Landau, Collected Papers of L.D. Landau, Ed. D. ter Haar, Pergamon, London 1965.

[9] M. Koralewski, J. Stankowska, T. Iglesias, J. Gonzalo, J. Phys., Condens. Matter 8, 4079 (1996).

[10] R. Clark, L. Benguigui, J. Phys. C, Solid State Phys. 10, 1963 (1977)

[11] J.L. Kirk, L.E. Cross, J.P. Dougherty, Ferroelectrics 11, 439 (1976).

[12] A.J. Miller, J. Penfold, G.D. Pitt, G.A. Saunders, in: High Pressure Science and Technology, Vol. 1, Eds. B. Vedar, Ph. Marteau, Pergamon Press, Oxford 1980, p. 503.

[13] M. Koralewski, A. Molak, J. Phys., Condens. Matter 11, 1341 (1999).

[14] V.N. Moiseenko, I.I. Peters, V.P. Sugonjak, V.G. Linnik, Fiz. Tverd. Tela 27, 2143 (1975).

[15] M. Połomska, M. Malinowski, H.H. Otto, Phys. Status Solidi A 56, 335 (1979). 
[16] H. Iwasaki, S. Miyazawa, H. Koizumi, K. Rugii, N. Niizeki, J. Appl. Phys. 43, 4907 (1972).

[17] S. Nanamatsu, H. Sugiyama, H. Doi, Y. Kondo, J. Phys. Soc. Japan 31, 616 (1971).

[18] I.A. Santos, J.A. Eiras, J. Phys., Condens. Matter 13, 11733 (2001).

[19] B.A. Strukow, E.W. Sinjakow, E.P. Majszczik, K.A. Minajewa, W.G. Monja, O.G. Vlokh, Izv. AN SSSR, Ser. Fiz. 41, 692 (1977).

[20] G.A. Samara, J. Phys. Soc. Japan Suppl. 28, 399 (1969). 